\title{
Carrier-wave Rabi flopping: role of the carrier-envelope phase
}

\author{
Oliver D. Mücke, Thorsten Tritschler, and Martin Wegener \\ Institut für Angewandte Physik, Universität Karlsruhe (TH), Wolfgang-Gaede-Strasse 1, 76131 Karlsruhe, Germany \\ Uwe Morgner \\ Max-Planck-Institut für Kernphysik, Saupfercheckweg 1, 69117 Heidelberg, Germany \\ Franz X. Kärtner \\ Department of Electrical Engineering and Computer Science and Research Laboratory of Electronics, \\ Massachusetts Institute of Technology, Cambridge, Massachusetts 02139 \\ Galina Khitrova and Hyatt M. Gibbs \\ Optical Sciences Center, University of Arizona, Tucson, Arizona 85721 \\ Received April 6, 2004

\begin{abstract}
Recently, a dependence of Rabi flopping on the carrier-envelope phase of the exciting laser pulses was predicted theoretically [Phys. Rev. Lett. 89, 127401 (2002)] for excitation of a thin semiconductor film with intense few-cycle pulses. Here, we report corresponding experiments on 50-100-nm thin GaAs films excited with 5-fs pulses. We find a dependence on the carrier-envelope phase arising from the interference of sidebands from the fundamental or the third-harmonic Mollow triplet, respectively, with surface second-harmonic generation. (C) 2004 Optical Society of America

OCIS codes: $190.7110,300.6470$
\end{abstract}

Nonlinear optics has recently entered a new era in which the phase between the rapidly oscillating carrier wave and the electric field envelope of a laser pulse, the carrier-envelope phase $\phi$, plays an important role. Several different schemes to measure $\phi$ itself have been proposed ${ }^{1-3}$ and realized experimentally. ${ }^{4-6}$ In 2002 carrier-wave Rabi flopping was proposed as an interesting candidate. ${ }^{7}$ The underlying physics is schematically shown in Fig. 1. For resonant excitation of a two-levelsystem-like transition, the Rabi frequency $\Omega_{R}(t)=$ $d \hbar^{-1} \tilde{E}(t) \cos \left(\omega_{0} t+\phi\right)=\Omega_{R} \cos \left(\omega_{0} t+\phi\right)$ modulates the transition frequency $\Omega=\omega_{0}$. Here, $d$ is the dipole matrix element, $\tilde{E}(t)$ is the electric field envelope, and $\omega_{0}$ is the carrier frequency. This modulation leads to sidebands around the fundamental as well as the third harmonic. Together with the central peaks, one obtains a fundamental Mollow triplet and a third-harmonic Mollow triplet with phases $\phi$ and $3 \phi$, respectively. When the condition $\Omega_{R} / \omega_{0}=1$ is approached, the high-energy sideband of the fundamental Mollow triplet interferes with the low-energy sideband of the third-harmonic Mollow triplet. The beat note with a difference phase of $2 \phi$ leads to a dependence on the carrier-envelope phase. Because of the underlying inversion symmetry, the sign of the electric field would remain undetermined. If, on the other hand, a second-order process (which was neglected in Ref. 7) with phase $2 \phi$ could be added, the difference phase would be $1 \phi$, and the sign of the electric field would matter (see ellipse in Fig. 1). A peculiarity of carrier-wave Rabi flopping is that, in principle, one gets a dependence on the carrier-envelope phase even for pulses containing many cycles of light. For strong-field excitation of atoms, a phase dependence in the multicycle regime has been discussed only recently. ${ }^{8}$

However, skepticism was expressed as to whether the above simple and intuitive picture of carrier-wave Rabi flopping would actually apply to semiconductor experiments in which the bands (rather than discrete levels), Coulomb correlations, renormalization effects, scattering, and dephasing complicate the situation. This has recently been clarified by a microscopic theory based on the semiconductor Bloch equations that accounts for all these aspects. ${ }^{9} \quad$ A comparison of calculated and measured third-harmonic spectra for single pulses showed good qualitative agreement, indicating that the above simple picture is indeed qualitatively correct.

In this Letter we present experiments on thin films of $\mathrm{GaAs}\left(E_{g}=1.42 \mathrm{eV}\right)$ revealing the anticipated dependence on the carrier-envelope phase under

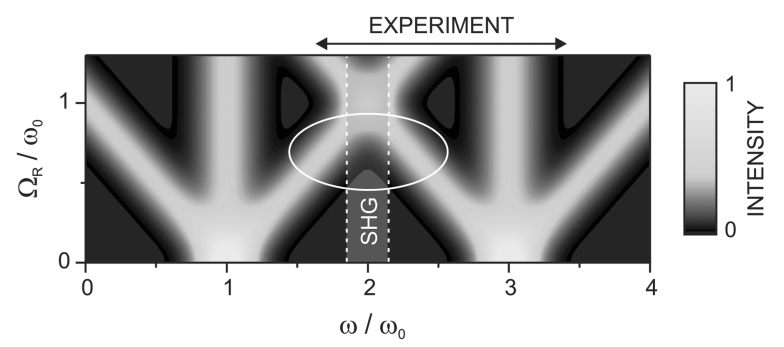

Fig. 1. Scheme of emission spectra arising from carrierwave Rabi flopping. The interference (see ellipse) of the two Mollow triplets and (or) surface second-harmonic generation (SHG) leads to a dependence on carrier-envelope phase $\phi$. 
conditions closely similar to those described in Ref. 7 . Following the suggestions of the theory, ${ }^{7}$ we employ thin GaAs layers of thickness $l$ (without AlGaAs barriers) directly grown on a sapphire substrate in a molecular-beam epitaxy machine. The growth of the GaAs layer on the 0.43-mm-thick, 51-mm-diameter epiready sapphire substrate (University Wafer) mounted in an In-free molyblock was performed the same as for a GaAs wafer with a growth rate of $0.25 \mathrm{~nm} / \mathrm{s}$. We investigated four samples with $l=25 \mathrm{~nm}$ (two samples), $l=50 \mathrm{~nm}$, and $l=100 \mathrm{~nm}$. Only the latter two turn out to have a damage threshold sufficiently large to actually perform the experiments at large Rabi energies. In our experiments 5 -fs linearly polarized optical pulses ${ }^{10}$ from a mode-locked laser oscillator are tightly focused onto the GaAs film through the air-GaAs interface (as opposed to the air-sapphire interface) by means of a high-numerical-aperture (N.A. 0.5) reflective microscope objective. This leads to a spot radius of approximately $1 \mu \mathrm{m}$. We estimate that an average laser power of $36.5 \mathrm{~mW}$ in front of the sample at a repetition frequency of $f_{r}=81 \mathrm{MHz}$ corresponds to a peak intensity of $I=2.8 \times 10^{12} \mathrm{~W} / \mathrm{cm}^{2}$. With dielectric constant $\epsilon=10.9$ and $d=0.5$ e nm for GaAs we further estimate a peak electric field of $\widetilde{E}_{0}=2.1 \times 10^{9} \mathrm{~V} / \mathrm{m}$ or a Rabi energy of $\Omega_{R} / \omega_{0}=0.76$ inside the GaAs film. To get to such high Rabi energies in the experiment without damaging the sample, we have already chopped the laser intensity at a frequency of approximately $100 \mathrm{~Hz}$ and with a duty cycle of approximately $3 \%$. The light emitted by the sample into the forward direction is collected by a second reflective microscope objective (N.A. 0.5), spectrally prefiltered and sent into a grating spectrometer.

To get an overview and to obtain insight into the dynamics, ${ }^{11}$ we excite the sample with a pair of pulses from a balanced and stabilized Michelson interferometer. ${ }^{12}$ The corresponding time delay of the two pulses is called $\tau$. For $\tau=0$ the two arms add constructively, leading to the largest Rabi energy. For increasing $|\tau|$ the effective Rabi energy gradually decreases. Hence the largest splitting in the optical spectra is expected for $\tau=0$. The laser intensities $I$ quoted in what follows refer to the total excitation intensity in front of the sample at time delay $\tau=0$. Figure 2 shows the spectra of light emitted into the forward direction for the $l=100 \mathrm{~nm}$ sample. Figure 2(a) corresponds to low excitation intensity $I$, and Fig. 2(b) corresponds to high excitation. The contribution centered around the 425-nm wavelength is due to surface SHG. This interpretation has been confirmed in independent experiments (not shown) in which $l$ has been varied. In Fig. 2(a) for $\tau=0$ an additional rather sharp peak around the third harmonic of the GaAs bandgap is observed $\left(3 E_{g}=4.26 \mathrm{eV}\right)$. At high excitation [Fig. 2(b)] this peak at $\tau=0$ splits into three, one of which overlaps with the surface SHG. The solid curves are guides to the eye and indicate that the splitting decreases with increasing $|\tau|$ as expected. These three peaks are interpreted as the third-harmonic Mollow triplet, an assignment that was recently confirmed by com- paring single-pulse experiments with solutions of the semiconductor Bloch equations. ${ }^{9}$ Because of bandgap renormalization the three peaks are redshifted with respect to the third harmonic and are no longer symmetrical around the center peak. Note also that a contribution from the fundamental moves into the picture from the top. Following our introduction this is expected to be the high-energy peak of the fundamental Mollow triplet. The data of the $l=50 \mathrm{~nm}$ sample (not shown) are compatible with those of the $l=100 \mathrm{~nm}$ sample.

We now come to the main result of this work. Figure 3 shows measured $\mathrm{rf}$ power spectra of the signals corresponding to Fig. 2(b). To enhance the signal levels, we removed the interferometer, leading to a larger average laser power of approximately $43 \mathrm{~mW}$ in front of the sample. In this set of experiments no chopping with a low duty cycle is used (it would be incompatible with using the rf spectrum analyzer). Compensating this increased power by slightly moving the GaAs film out of focus results in similar light intensities in the GaAs sample, and hence in similar Rabi energies, but leads to larger absolute signal levels due to the increased area of emitting GaAs. This trick boosts the signal levels in the rf power spectrum upward, which is essential considering the peak heights in Fig. 3. In these experiments we employed a second optical grating spectrometer (Jobin-Yvon HR460 with a 300-line/mm grating
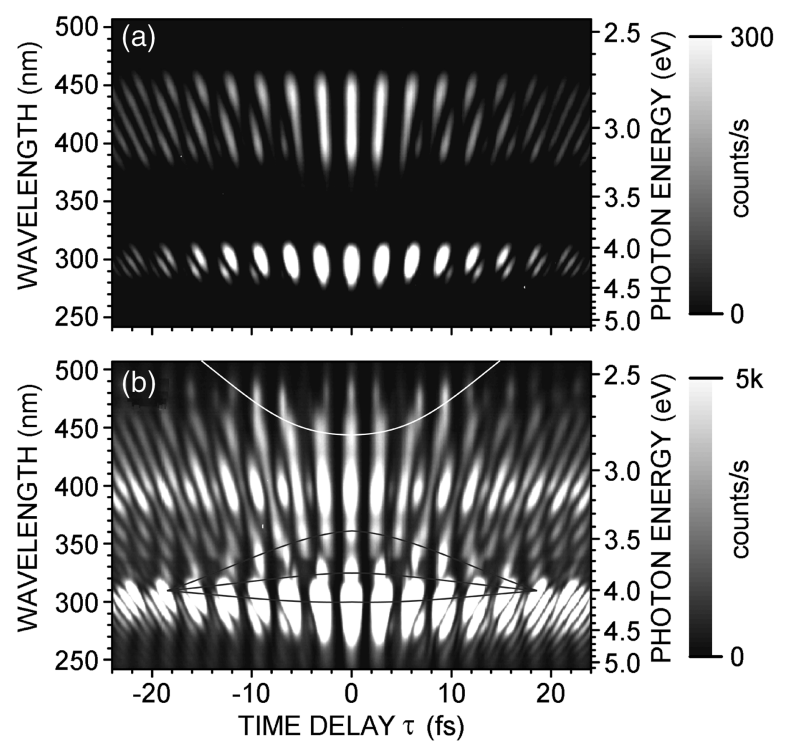

Fig. 2. Emission spectra of an $l=100 \mathrm{~nm}$ thin GaAs film for excitation with a pair of 5 -fs pulses with time delay $\tau$. Excitation intensity (a) $I=0.24 \times 10^{12} \mathrm{~W} / \mathrm{cm}^{2}$, (b) $I=2.8 \times 10^{12} \mathrm{~W} / \mathrm{cm}^{2}$. The contribution centered on the 425-nm wavelength is due to surface SHG. The single peak in (a) centered around the $300-\mathrm{nm}$ wavelength (the third harmonic of the GaAs bandgap) evolves into three peaks in (b), which are attributed to the carrier-wave Mollow triplet. The corresponding three black curves are a guide to the eye. The white curve at the top (another guide to the eye) indicates the position of the high-energy peak of the fundamental Mollow triplet. For (b) we estimate that the peak Rabi energy inside the GaAs film is given by $\Omega_{R} / \omega_{0}=0.76$. 


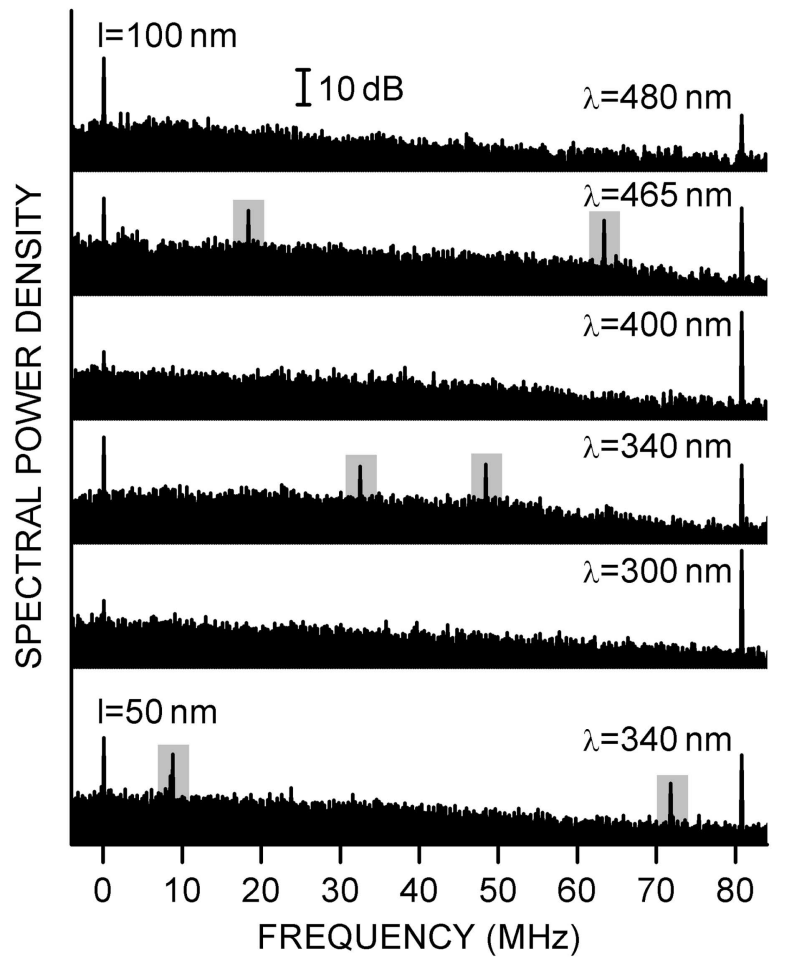

Fig. 3. Experiment: rf power spectra (logarithmic scale), $10-\mathrm{kHz}$ resolution and video bandwidth, for various optical detection wavelengths $\lambda$ and two GaAs film thicknesses $l$ as indicated. The peaks at the carrier-envelope frequency $f_{\phi}$ and at $\left(f_{r}-f_{\phi}\right)$ are highlighted by gray areas.

blazed at a 250 -nm wavelength). Opening both slits of this spectrometer to a width of $2 \mathrm{~mm}$ corresponds to detection of a $20-\mathrm{nm}$ broad spectral interval with center wavelength $\lambda$. The exit slit of the spectrometer is connected to a photomultiplier tube (Hamamatsu R4332, bialkali photocathode). Its output voltage is fed into a rf spectrum analyzer (Agilent PSA E4440A) operated at a $10-\mathrm{kHz}$ resolution and video bandwidth. In Fig. 3 selected examples are shown. The peak at $81 \mathrm{MHz}$ arises from repetition frequency $f_{r}$ of the laser oscillator. At the wavelengths of $\lambda=465 \mathrm{~nm}$ and $\lambda=340 \mathrm{~nm}$ in Fig. 3 we find the largest peaks at the carrier-envelope frequency $f_{\phi}$ and at the difference frequency $\left(f_{r}-f_{\phi}\right)$. This corresponds to optimum interference of the high-energy fundamental Mollow sideband with the surface SHG and optimum interference of the surface SHG with the low-energy third-harmonic Mollow sideband, respectively. Note that for these wavelengths the $f_{\phi}$ and $\left(f_{r}-f_{\phi}\right)$ peaks in the $\mathrm{rf}$ power spectrum are less than $8 \mathrm{~dB}$ smaller than the $f_{r}$ peak, indicating that the relative modulation depth of the beat signal versus time is as large as $40 \%$. On the other hand, for wavelengths of $\lambda=480$, $400 \mathrm{~nm}$, and $300 \mathrm{~nm}$, respectively, no significant peak at $f_{\phi}$ occurs, even though the absolute signal levels are larger (see larger $f_{r}$ peak). This overall situation is consistent with the optical spectra shown in Fig. 2. The value of $f_{\phi}$ changes between the $\mathrm{rf}$ power spectra. This is mainly because we intentionally moved the intracavity prism near the high reflector to demonstrate the influence of intracavity dispersion on the results. Similar results are observed for the $l=50 \mathrm{~nm}$ thin sample (see lowest data set in Fig. 3).

In conclusion we have, for the first time to our knowledge, observed a dependence on the carrierenvelope phase resulting from the carrier-wave Mollow triplet in experiments on $50-100-\mathrm{nm}$ thin GaAs films excited with 5 -fs pulses. Assuming that the carrier-envelope frequency has already been stabilized to zero by some other means, this effect could be used to measure the carrier-envelope phase itself (at the sample location) despite the fact that the signals are rather small. This would require an appropriate calibration and takes advantage of the fact that the theory ${ }^{7}$ predicts only a minor variation of the carrier-envelope phase within such thin GaAs films. Carrier-wave Rabi flopping is unique in the sense that, in principle, one would expect an appreciable dependence on the carrier-envelope phase even for pulses containing many cycles of light.

The work of M. Wegener was supported by projects DFG-We 1497/11-1 and DFG-We 1497/9-1, that of U. Morgner by project DFG-Mo 850/2-1, and that of F. X. Kärtner by the Office of Naval Research and National Science Foundation grant ECS-0217358. H. M. Gibbs and G. Khitrova thank the National Science Foundation Atomic, Molecular, and Optical Physics for support. T. Tritschler's e-mail address is thorsten.tritschler@physik.uni-karlsruhe.de.

\section{References}

1. A. de Bohan, P. Antoine, D. B. Milošević, and B. Piraux, Phys. Rev. Lett. 81, 1837 (1998).

2. C. Lemell, X.-M. Tong, F. Krausz, and J. Burgdörfer, Phys. Rev. Lett. 90, 076403 (2003).

3. D. B. Milošević, G. G. Paulus, and W. Becker, Opt. Express 11, 1418 (2003), http://www.opticsexpress.org.

4. A. Baltuška, Th. Udem, M. Uiberacker, M. Hentschel, E. Goulielmakis, Ch. Gohle, R. Holzwarth, V. S. Yakovlev, A. Scrinzi, T. W. Hänsch, and F. Krausz, Nature 421, 611 (2003).

5. G. G. Paulus, F. Lindner, H. Walther, A. Baltuška, E. Goulielmakis, M. Lezius, and F. Krausz, Phys. Rev. Lett. 91, 253004 (2003).

6. A. Apolonski, P. Dombi, G. G. Paulus, M. Kakehata, R. Holzwarth, Th. Udem, Ch. Lemell, K. Torizuka, J. Burgdörfer, T. W. Hänsch, and F. Krausz, Phys. Rev. Lett. 92, 073902 (2004).

7. O. D. Mücke, T. Tritschler, M. Wegener, U. Morgner, and F. X. Kärtner, Phys. Rev. Lett. 89, 127401 (2002).

8. G. Sansone, C. Vozzi, S. Stagira, M. Pascolini, L. Poletto, P. Villoresi, G. Tondello, S. De Silvestri, and M. Nisoli, Phys. Rev. Lett. 92, 113904 (2004).

9. Q. T. Vu, H. Haug, O. D. Mücke, T. Tritschler, M. Wegener, G. Khitrova, and H. M. Gibbs, Phys. Rev. Lett. 92, 217403 (2004).

10. U. Morgner, F. X. Kärtner, S. H. Cho, Y. Chen, H. A. Haus, J. G. Fujimoto, E. P. Ippen, V. Scheuer, G. Angelow, and T. Tschudi, Opt. Lett. 24, 411 (1999).

11. J. Tate and D. Schumacher, Phys. Rev. Lett. 87, 053901 (2001).

12. M. U. Wehner, M. H. Ulm, and M. Wegener, Opt. Lett. 22, 1455 (1997). 